\title{
Prediction of Acute Renal Failure in Dengue Fever Patients
}

\author{
Salwa Abd Almoneim Mohammed Ali1,2*, Mohammed Omer Abaker Gibreel3, \\ Nazik Sir El Khatim Bakhit Suliman', Ali Khider Ali Mohammed4, Bakri Yousif Mohamed Nour²
}

\author{
${ }^{1}$ Department of Clinical Chemistry, Eastern Sudan College of Medical Science and Technology, Port Sudan, Sudan \\ ${ }^{2}$ Department of Clinical Chemistry, Faculty of Medical Laboratory Sciences, University of Gezira, Wad Medani, Sudan \\ ${ }^{3}$ Department of Hematology and Immunohematology, Port Sudan Ahlia College, Port Sudan, Sudan \\ ${ }^{4}$ Department of Microbiology, Eastern Sudan College of Medical Science and Technology, Port Sudan, Sudan \\ Email: ^salwamoneim64@gmail.com,m_omer8164@yahoo.com, naziksuliman18@gmail.com, bakrinour@gmail.com
}

How to cite this paper: Ali, S.A.A.M., Gibreel, M.O.A., Suliman, N.S. El K.B., Mohammed, A.K.A. and Nour, B.Y.M. (2022) Prediction of Acute Renal Failure in Dengue Fever Patients. Open Journal of Urology, 12, 99-106.

https://doi.org/10.4236/oju.2022.122010

Received: December 13, 2021

Accepted: February 7, 2022

Published: February 10, 2022

Copyright (c) 2022 by author(s) and Scientific Research Publishing Inc. This work is licensed under the Creative Commons Attribution International License (CC BY 4.0).

http://creativecommons.org/licenses/by/4.0/

\begin{abstract}
Background: Dengue virus (DENV) infection is caused by an arboviral strain and is transmitted by the mosquito Aedes aegypti which is found in Sudan especially Red Sea and Kassala states in the east. The disease is known to cause renal disturbances and a thorough understanding of that will potentially help in the prediction, diagnosis and treatment of the disease. Methods: This study is a prospective observational cross sectional study conducted in the Eastern Sudan College of Medical Science and Technology and Port Sudan Teaching Hospital. 200 confirmed Dengue virus infected patients along with 200 healthy appearing adults (control) were enrolled for the study. Statistical analysis was carried out after the collection of patients' demographic, clinical, and investigational data including serum urea and creatinine values. Ethical approval was obtained from the ministry of health, Red Sea state and informed written consent was obtained from each participant. Results: The highest incidence of DENV infection was observed in individuals of the middle age group (29\%). Elevated blood urea levels were detected in $10(5 \%)$ patients while elevated creatinine levels were seen in 17 (8.5\%) patients. Although fallen within reference ranges found in the literature, mean blood urea and creatinine values differed significantly between patients and controls and between different categories of the disease. Mean blood urea concentration showed a statistically significant difference between the control (22.3 $\mathrm{mg} / \mathrm{dl})$ and the test $(28.4 \mathrm{mg} / \mathrm{dl})(\mathrm{P}$ value $<0.001)$. Similarly, mean serum creatinine in the control $(0.70 \mathrm{mg} / \mathrm{dl})$ differed significantly ( $\mathrm{P}$ value $<0.001)$ from test $(0.94 \mathrm{mg} / \mathrm{dl})$. Clinically, fever was present in $97.5 \%$ of patients, headache in $95.5 \%$, joints pain in $71 \%$, lethargy in $67 \%$, vomiting in $49 \%$, skin rash in $40 \%$, abdominal pain in $24 \%$ and bleeding in $17.5 \%$. Conclusion: We strongly conclude that renal involvement is not uncommon in Dengue fever
\end{abstract}


and that blood urea and creatinine evaluation should be considered in the counseling of DENV infection patients. Patients need to be subjected to necessary laboratory investigations associated with acute kidney injury to decrease the rate of morbidity and mortality associated with the disease.

\section{Keywords}

Dengue Virus, Acute Kidney Injury, Hemorrhagic Fever, Shock Syndrome, Eastern Sudan

\section{Introduction}

Dengue fever is a mosquito borne viral infection that constitutes a major health problem in tropical and sub-tropical regions of the world. Urbanization and air travel are among the factors associated with the increased trend of infection in recent years. Over 2.5 billion people of the world's population are now at risk for Dengue. The consequence of Dengue virus (DENV) infection ranges from the asymptomatic condition (Dengue fever (DF), to more severe forms, such as Dengue hemorrhagic fever (DHF) and Dengue shock syndrome (DSS). Severe Dengue is characterized either by plasma leakage, fluid accumulation, respiratory distress, severe bleeding, or organ impairment. Clinical manifestations offer the earliest markers in predicting severe Dengue Disease [1].

Patients with Dengue fever are clustered into two groups: one with warning signs including abdominal pain, mucosal bleeding and hepatomegaly that warrant ICU admission and the other without those signs. Early prediction of severe Dengue infection in patients without any warning signs who may later develop severe DHF is very important to give the best supportive care since approved vaccines for immunization are yet to be commercialized. An ideal biomarker should be able to identify individuals who are at risk of developing severe Dengue infection [2]. Dengue patients show fever symptoms during peak of viraemia while DHF/DSS appears during the time when the virus has been cleared from the circulation suggesting that severe Dengue disease is most likely associated with immunopathology [2].

Dengue infection has been associated with a variety of renal disorders. Acute renal failure is a potential complication of severe Dengue infection and is typically associated with hypotension, rhabdomyolysis, or hemolysis. Acute renal failure occasionally complicates severe Dengue infection and carries a high mortality rate. Transient proteinuria has been detected in most patients with severe Dengue infection. Hematuria has been reported in a significant subset of patients with severe forms of the infection. Various types of glomerulonephritis have been reported during or shortly after Dengue infection in humans and mouse models. Meningeal proliferation and immune complex deposition are the dominant histologic features. On a rare occasion, Dengue infection is associated with systemic autoimmune disorders involving the kidneys. In the vast majority 
of cases, Dengue infection and associated renal disorders are self-limited [3].

Dengue infection evolving into systemic lupus erythematosus and lupus nephritis has been reported with antibodies directed against nuclear antigens including ANA and double-stranded DNA [3].

In Sudan, Dengue fever (DF) is considered a major public health issue in the Eastern region of the country, where it has been reported since 1908 with endemicity and frequent outbreaks in the coastal and sub-coastal areas of the Red Sea and Kassala states. Dengue fever reported in the Sudanese population is associated with serious presentations including DHF and DSS with an increased rates of morbidity and mortality. Acute kidney injury (AKI) is a serious and potentially lethal complication of this disease. It is obvious that Dengue Fever is becoming a serious problem and this needs specific investigation and diagnosis to avoid complications of the disease. Approximately 2.5 billion individuals from more than 110 countries are in danger of DENV infection each year due to the lack of an efficient vaccine and a specific treatment [4]. Dengue fever is transmitted by Aedes aegypti with other species of Aedes mosquito involved [5]. There are 4 serotypes of dengue viruses known as DENV-1, DENV-2, DENV-3, and DENV-4 [6].

Renal function is routinely assessed by a profile that includes the determination of serum urea, creatinine, creatinine clearance and electrolytes. An elevated concentration of urea in the blood is called azotemia. Very high plasma urea concentration accompanied by renal failure is called uremia, or the uremic syndrome. This condition is eventually fatal if not treated by dialysis or transplantation [7]. Decreased renal function causes an increase in plasma urea concentration as a result of compromised urea excretion. Renal causes of elevated urea include acute and chronic renal failure, glomerular nephritis, tubular necrosis, and other intrinsic renal diseases. Measurement of creatinine concentration is used to determine sufficiency of kidney function and the severity of kidney damage and to monitor the progression of kidney disease. The amount of creatinine in the bloodstream is reasonably stable, although the protein content of the diet does influence the plasma concentration because of the constancy of endogenous production [8].

Acute kidney injury (AKI) is a condition where kidneys suddenly stop working properly. It can range from minor loss of kidney function to complete kidney failure. AKI normally happens as a complication of another serious illness. It is not the result of a physical blow to the kidneys, as the name might suggest. This type of kidney damage is usually seen in older people who are unwell with other conditions and the kidneys are also affected. It is essential that AKI is detected early and treated promptly. Without quick treatment, abnormal levels of salts and chemicals can build up in the body, which affects the ability of other organs to work properly. If the kidneys shut down completely, this may require temporary support from a dialysis machine, or lead to death [9] [10]. This study is designed to incorporate the prediction of acute renal injury as a routine check in approaching Dengue fever patients. 


\section{Methods}

This was an hospital-based cross sectional descriptive study conducted at Port Sudan Teaching Hospital in the period from June 2018 to September 2021 to evaluate the involvement of acute kidney injury (where kidney suddenly stops working properly) in confirmed Dengue fever patients. Enrolled in the study are 200 diagnosed Dengue fever patients (fixed number) and other 200 healthy appearing people as a control group. Patients with a past history of renal or liver disease before diagnoses of Dengue fever and patients who have lupus erythromatosis were excluded. Ethical approval was achieved from the research committee in the ministry of health, Red Sea state and an informed written consent was obtained from each participant.

Venous blood samples were collected from each patient, one in heparinized containers, and another in plain containers then refrigerated at $2^{\circ} \mathrm{C}-8^{\circ} \mathrm{C}$ till analyzed. The diagnosis of Dengue fever was established by the detection of DENV IgG/IgM antibodies by ELISA Technique.

Blood urea and creatinine were measured photometrically by the enzymatic method using the PT350 Bio system spectrophotometer and reagent kits from Biosystems Company. Data were statistically analyzed by the SPSS software and frequencies, concentrations and correlations were illustrated.

\section{Results}

The higher percentage of patients of DENV infection was from individuals of middle age (40 - 49 years old) representing $29 \%$ of cases (Figure 1).

Out of the 200 confirmed DENV positive patients included in this study, 126 (63\%) were males and 74 (37\%) were females along with other 200 healthy appearing adults of whom $100(50 \%)$ were males and $100(50 \%)$ were females as a control group, 160 (80\%) were found to suffer from mild Dengue fever, 35 (17.5\%) suffered from Dengue hemorrhage fever, 5 (2.5\%) suffered from Dengue shock syndrome. Eight patients (4\%) showed signs of acute kidney injury (1 patient with acute kidney injury the result of ANA, anti-dsDNA were significantly increased).

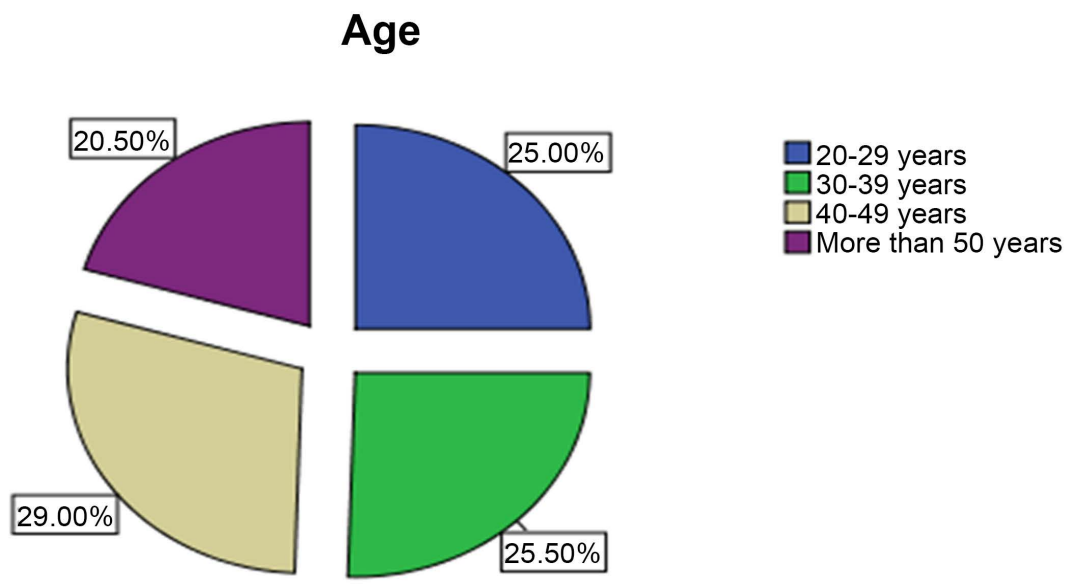

Figure 1. Age distribution (in years) in patients. 
Table 1 shows a statistically significant difference between Dengue fever patients and the control group in the concentration of serum urea and creatinine.

Bio chemical findings in the different categories of Dengue virus infection showed statistically significant differences. Table 2 illustrates the difference between Dengue fever and Dengue hemorrhagic fever.

Similarly, Table 3 shows a significant difference in biochemical finding between Dengue fever and Dengue shock syndrome especially in urea concentration.

In contrast, Table 4 illustrates an insignificant difference in biochemical finding between Dengue hemorrhagic fever and Dengue shock syndrome.

Insignificant differences were also seen in biochemical findings according to gender in Dengue virus infected patients in different categories of the disease in urea and creatinine concentration apart from creatinine in males and females of Dengue fever (Tables 5-7).

\section{Discussion}

Dengue fever can be presented with a variety of clinical presentations associated

Table 1. Comparison between test and control in biochemical findings.

\begin{tabular}{ccccc}
\hline \multirow{2}{*}{ Parameter } & Reference & Test (Mean \pm 1 SD) & Control (Mean \pm 1 & \multirow{2}{*}{ P value } \\
& Range & $\mathbf{N}=200$ & SD) $\mathbf{N}=200$ & \\
\hline Urea $(\mathrm{mg} / \mathrm{dl})$ & $15-50$ & $28.40 \pm 25.7$ & $22.30 \pm 6.30$ & $<0.001$ \\
Creatinine $(\mathrm{mg} / \mathrm{dl})$ & Up to 1 & $0.94 \pm 0.08$ & $0.70 \pm 0.15$ & $<0.001$ \\
\hline
\end{tabular}

Table 2. Comparison between DF and DHF in biochemical findings.

\begin{tabular}{cccc}
\hline Parameter & DF (Mean \pm 1 SD) & DHF (Mean \pm 1 SD) & P value \\
& $\mathrm{N}=160$ & $\mathrm{~N}=35$ & \\
\hline Urea (mg/dl) & $22.3 \pm 6.005$ & $44.88 \pm 39.80$ & 0.020 \\
Creatinine (mg/dl) & $0.75 \pm 0.18$ & $1.44 \pm 1.45$ & $<0.001$ \\
\hline
\end{tabular}

Table 3. Comparison between DF and DSS in biochemical findings.

\begin{tabular}{cccc}
\hline Parameter & DF (Mean \pm 1 SD) & DSS (Mean \pm 1 SD) & P value \\
& $\mathbf{N}=160$ & $\mathbf{N}=\mathbf{5}$ & \\
\hline Urea $(\mathrm{mg} / \mathrm{dl})$ & $22.3 \pm 6.005$ & $109.30 \pm 76.50$ & $<0.001$ \\
Creatinine $(\mathrm{mg} / \mathrm{dl})$ & $0.75 \pm 0.18$ & $3.54 \pm 1.10$ & 0.050 \\
\hline
\end{tabular}

Table 4. Comparison between DHF and DSS in biochemical findings.

\begin{tabular}{cccc}
\hline Parameter & $\begin{array}{c}\text { DHF (Mean } \pm 1 \text { SD) } \\
\mathrm{N}=35\end{array}$ & $\begin{array}{c}\text { DSS (Mean } \pm 1 \mathrm{SD}) \\
\mathrm{N}=\mathbf{5}\end{array}$ & P value \\
\hline Urea $(\mathrm{mg} / \mathrm{dl})$ & $44.88 \pm 39.80$ & $109.3 \pm 76.5$ & 0.500 \\
Creatinine $(\mathrm{mg} / \mathrm{dl})$ & $1.44 \pm 1.45$ & $3.54 \pm 1.10$ & 0.100 \\
\hline
\end{tabular}


Table 5. Comparison between dengue fever male and female patients in biochemical findings.

\begin{tabular}{cccc}
\hline Parameter & Males (Mean $\pm 1 \mathrm{SD})$ & Females (Mean $\pm \mathbf{1 ~ S D})$ & P value \\
& $\mathrm{N}=\mathbf{1 0 3}$ & $\mathrm{N}=\mathbf{5 7}$ & \\
\hline Urea $(\mathrm{mg} / \mathrm{dl})$ & $22.92 \pm 6.20$ & $21.21 \pm 5.60$ & 0.080 \\
Creatinine $(\mathrm{mg} / \mathrm{dl})$ & $0.77 \pm 0.19$ & $0.70 \pm 0.11$ & 0.040 \\
\hline
\end{tabular}

Table 6. Biochemical findings in patients of dengue hemorrhagic fever based on gender.

\begin{tabular}{cccc}
\hline Parameter & Males (Mean \pm 1 SD) & Females (Mean \pm 1 SD) & P value \\
& $\mathbf{N}=\mathbf{2 0}$ & $\mathbf{N}=\mathbf{1 5}$ & \\
\hline Urea (mg/dl) & $53.5 \pm 49.70$ & $33.4 \pm 15.70$ & 0.100 \\
Creatinine (mg/dl) & $1.70 \pm 1.85$ & $1.05 \pm 0.45$ & 0.200 \\
\hline
\end{tabular}

Table 7. Biochemical findings in patients of dengue shock syndrome based on gender.

\begin{tabular}{cccc}
\hline Parameter & Males (Mean \pm 1 SD) & Females (Mean \pm 1 SD) & P value \\
& $\mathbf{N}=\mathbf{3}$ & $\mathbf{N}=\mathbf{2}$ & \\
\hline Urea $(\mathrm{mg} / \mathrm{dl})$ & $156.0 \pm 52.40$ & $39.25 \pm 39.24$ & 0.070 \\
Creatinine $(\mathrm{mg} / \mathrm{dl})$ & $3.90 \pm 0.18$ & $3.10 \pm 0.21$ & 0.400 \\
\hline
\end{tabular}

with unpredictable progression and outcomes. They range from asymptomatic illness to severe shocks eventually resulting in death [11]. Dengue is considered a major health threat by the World Health Organization. An increase in infection has been seen in recent years due to many factors including urbanization and air travel. Approximately over 2.5 billion people of the words population are now at risk for Dengue [12]. The purpose why only a few DENV disease cases get worse is poorly understood but the host immune response has been considered as the major factor responsible for Dengue pathogenesis [13]. Dengue infection is associated with multiple organ dysfunction involving liver, muscles, heart, brain and kidney [14]. Dengue fever has been associated with various types of renal disorders such as proteinuria, hematuria, glomerulonephritis, and acute kidney injury [15].

In this study, out of the confirmed two hundred Dengue patients, eight patients (4\%) had Acute Kidney Injury (AKI). Results that are similar to those of $\mathrm{s}$ study done by Warappa ES. et al. who reported that the prevalence of renal manifestation in Dengue fever patients was (9.02\%) [16]. The fact that the prevalence of DF in males was higher than females in our study along with the clinical presentation that fever was detected in (97.5\%) of patients, is similar to the results of a study done by Relwani P. R. et al. who found that fever was one of the common features associated with Dengue infection (98.66\%) [17].

In our study the (mean $\pm 1 \mathrm{SD}$ ) of serum urea levels of $(22.3 \pm 6.01 \mathrm{mg} / \mathrm{dl})$ for Dengue fever patients, $(44.88 \pm 39.8 \mathrm{mg} / \mathrm{dl})$ for Dengue hemorrhagic fever patients, and $(109.3 \pm 76.5 \mathrm{mg} / \mathrm{dl})$ for Dengue shock syndrome patients, was simi- 
lar to what was seen by Guzman MG. and Halsted SB. [18] who showed increased urea levels in all categories of the disease.

Similarly, the (mean $\pm 1 \mathrm{SD}$ ) of creatinine levels in Dengue fever patients was $(0.75 \pm 0.18 \mathrm{mg} / \mathrm{dl})$, in Dengue Hemorrhagic fever patients was $(1.44 \pm 1.45$ $\mathrm{mg} / \mathrm{dl})$, and in Dengue shock syndrome was $(3.5 \pm 1.10 \mathrm{mg} / \mathrm{dl})$, is agreed to the results of a study by Taurqeer H. and Amer K. [19] who reported an increase in creatinine levels in all the categories of Dengue infection.

The present study found that only one patient with acute kidney injury had increased levels of anti-dsDNA, and ANA which is similar to the findings of a study by Rajadhyaksha A. and Mehra S. in a case report [20] showing an increase in anti-dsDNA and ANA.

\section{Conclusion}

Renal involvement is frequent in patients with Dengue fever especially in those exhibiting complications of the disease. Routine assessment of these patients should include the determination of blood urea and creatinine as a check marker for the prediction of acute kidney injury.

\section{Acknowledgements}

We would like to thank many people without the help of whom this work would have not seen light. Thanks are for our families for valuable support. Especial thanks are for Dr. Monammed Haroun Mohammed Ali the head manager of the department of training, Red Sea state for his unlimited encouragement and support.

\section{Conflicts of Interest}

The authors have no conflicts of interest to disclose.

\section{References}

[1] John Wiley and Sons Ltd. (2017) Regulatory T-Cells in Acute Dengue Viral Infection. The Journal of Cells Molecules System and Technologies, 26, 162.

[2] John, D.V., Lin, Y.S. and Perng, G.C. (2015) Biomarker of Sever Dengue Disease: A Review. Journal of Biomedical Science, 22, 83. https://doi.org/10.1186/s12929-015-0191-6

[3] Lizarraga, K.J. and Nayer, A. (2014) Dengue Associated Kidney Disease. Journal of Nephropathology, 3, 57-62.

[4] Hernández, S., Puerta-Guardo, H., Aguilar, H., et al. (2016) Primary Dengue Virus Infections Induce Differential Cytokine Production in Mexican Patients. Memorias do Instituto Oswaldo Cruz, 111, 161-167. https://doi.org/10.1590/0074-02760150359

[5] Ahmed, A., Elduma, A., Magboul, B., Higazi, T. and Ali, Y. (2019) The First Outbreak of Dengue Fever in Greater Darfur Western Sudan. Tropical Medicine of Infectious Diseases, 4, 43. https://doi.org/10.3390/tropicalmed4010043

[6] Soe, H.J., Yong, B.Y.K., Jamil Al-Obaidi, M.M., Raju, C.S., Gudimella, R., Manikam, R., Sekaran, S.D., et al. (2018) Identifying Protein Biomarkers in Predicting Disease 
Severity of Dengue Virus Infection Using Immune-Related Protein Microarray. Journal Medicine, 97, 5. https://doi.org/10.1097/MD.0000000000009713

[7] Daher, E.D.F., Silva Junior, G.B., Vieira, A.P.F., et al. (2014). Acute Kidney Injury in a Tropical Country: A Cohort Study of 253 Patients in an Infectious Diseases Intensive Care Unit. Revista da Sociedade Brasileira de Medicina Tropical, 47, 86-89. https://doi.org/10.1590/0037-8682-0223-2013

[8] Gounden, V., Bhatt, H. and Jialal, I. (2021) Renal Function Tests.

[9] Doi, K., Yuen, P.S., Eisner, C., Hu, X., Leelahavanichkul, A., Schnermann, J. and Star, R.A. (2019) Reduced Production of Creatinine Limits Its Use as Marker of Kidney Injury in Sepsis. Journal of the American Society of Nephrology, 20, 1217-1221. https://doi.org/10.1681/ASN.2008060617

[10] Ahmed, M. and Akhanaizi (2018) Acute Kidney Injury and Continuous Renal Replacement Therapy in Children; What Pediatricians Need to Know. Korean Journal of Pediatrics, 61, 339.

[11] Relwani, P.R., Redkar, N.N. and Garg, D. (2019) Study of Electrolytes in Patients of Dengue in a Tertiary Care Hospital in India. International Journal of Advances in Medicine, 6, 763-768. https://doi.org/10.18203/2349-3933.ijam20192114

[12] Narayanan, M., Aravind, M.A., Thilothammal, N., Prema, R. and Ramanrty, N. (2002) Dengue Fever Epidemic in Chennai-A Study of Clinical Profile and Outcome. Indian Pediatrics, 39, 1027-1033.

[13] Vuong, N., Manh, D.H., Mai, N.T., Luong, V.I., Quan, V.D., Van Thuong, N., et al. (2016) Criteria of Persistent Vomiting the WHO 2009 Warning Signs for Dengue Case Classification. Tropical Medicine and Health, 44, 14. https://doi.org/10.1186/s41182-016-0014-9

[14] Unniklishnam, R., Faizal, B.P., Vijaya Kumar, P., Panl, G. and Sharma, R.N. (2015) Clinical and Laboratory Profile of Dengue in the Elderly. Journal of Family Medicine and Primary Care, 4, 369-378. https://doi.org/10.4103/2249-4863.161323

[15] Mekmullica, J., Suwanphatra, A., Thienpaitoon, H., Chansongsaknl, T., et al. (2005) Serum and Urine Sodium Levels in Dengue Patients. The Southeast Asian Journal of Tropical Medicine and Public Health, 36, 197-199.

[16] Lumpaopong, A., Kaewplang, P., Watananeeradej, V., Thirakhupt, P., Channanuanakit, J.S. and Srisuwan, K. (2010) Electrolyte Disturbances and Urine Analysis in Children with Dengue Infection. The Southeast Asian Journal of Tropical Medicine and Public Health, 41, 72-76.

[17] Widodo, D., Setiewan, B., Chenk, N. and Santoso, W.B. (2018) The Prevalence of Hypokalemia in Hospitalized Patients with Infection Disease Problem at Cipto Mangunkusumo Hospital, Jarkatra. Acta Medica Indonesiana, 38, 202-205.

[18] Guzman, M., Halstead, S., Artsob, H., et al. (2010) Dengue: A Continuing Global Threat. Nature Reviews Microbiology, 11, 7-16. https://doi.org/10.1038/nrmicro2460

[19] Khan, A.H. and Mallhi, T.H. (2016) Dengue Induced Nephropathies. SM Group; Chronic Kidney Disease. SM Journal.

[20] Rajadnyaksha, A. and Mehra, S. (2014) Dengue Fever Evolving into Systemic Lupus Erythromatosus and Lupus Nephritis: A Case Report. Lupus, 21, 999-1002.

http://www.Sagepublications.com https://doi.org/10.1177/0961203312437807 